\title{
Globalization to a Developing Economy: a Pain or a Gain
}

\author{
Roseline Oluitan PhD \\ Department of Accounting \& Finance Lagos State University, Ojoo, Lagos \\ Tel: 234-818-482- 3668 E-mail: roselinetoyin@yahoo.com
}

Received: November 23, 2013 Accepted: December 8, 2013

doi:10.5296/ber.v4i1.4603 URL: http://dx.doi.org/10.5296/ber.v4i1.4603

\begin{abstract}
This paper examines the concept of globalization and analyse the effect on both developed and developing economies to ascertain the actual impact on both economies. Arguments of supporters and opponents of the concept were discussed and empirical data discussed to arrive at who is actually benefitting from the concept. It observes that the principles on which the concept is based is not favourable to the developing countries for obvious reasons.

The study supports the view that there are loopholes in the processes which is being abused by mostly the multinationals to exploit the developing countries. It concludes that the concept is capable of positive impact, but will need to be modified by promoting institutions to turn the pain of the developing countries to gain.
\end{abstract}

\section{Introduction}

Globalization is one of the topical issues in the world today. It entails the growing integration of economies and societies around the world. To a lot of people, it has been a vital means to sustainable development mostly for third world economies hence one of the good things that has ever happened in the past few centuries. Some people vehemently oppose this view. They rather see it as a window dressing of the erstwhile slave trade by the developed countries. This school of thought is of the opinion that nothing good has ever happened to the developing countries through globalization, and if anything, it is largely insignificant to the gains that are reaped by the developed countries.

One thing is very clear about globalization and that is what it is impinged upon, put in other words, what it is targeted at in any economy. These according to Yusuf (2001) are:

- Growth of trade

- Capital flows and financial capability

- Easy migration 
- Information Technology and the Web

- Diffusion of Technology

According to him, all parts of the world are affected by globalization though these channels, but it is important to remember that the full force of change is felt by a relatively small number of upper and middle income countries due mainly to the level of integration into the global system

The key institutions promoting globalization are the World bank, International Monetary Fund (IMF) and World Trade Organization (WTO) who are interested in enhancing the standard of living of the world populace (eradicating poverty), improving bilateral trade and free movement towards enhanced knowledge and information flow. According to Rosenana (1997), globalization can be defined as "a label that is presently in vogue to account for peoples: Activities, Norms, Areas, Goods, services and Currencies that are decreasingly confined to a particular geographic space with local and established practices". It is imperative to state that the concept of globalization entails both microeconomic which refers to the technological revolution and its impact on the firm's leverage and macroeconomic aspect which focuses on the integration of markets for goods and services

In the course of this paper we shall look into arguments of the two groups, evidences that abound and take a position on the role of globalization to the developing economies, whether it has been a pain that further plunges them into further problems/ crises or that it has been a gain that has strived to improve the populace in this areas of focus.

\section{Effect of Globalization on the Development Countries}

In order to harness the effect of globalization on the developing countries, we shall consider critically three of the five key policy areas earlier enumerated which has direct relevance to finance and upon which globalization is impugned upon. The choice of the policy area is based on available data to justify the argument. These are.

\section{a) Growth of Trade and Capital flows}

One of the primary objectives of globalization is the promotion of free trade and open market which enhances global free competition amongst industries. It allows companies and firms to undertake voluntary economic transaction through cross border activities covering International Trade, Direct Foreign Investment and International Capital Flows. Thus De-Soysa \& Vadlamannati (2011) observes positive effects of the various forms of globalization and government respect for basic human rights for a sample of 118 countries and a sub-sample of developing countries thereby suggesting that globalization predicts better human rights. Similarly, Dreher et al (2010) postulates that physical integrity and rights significantly and robustly increase with globalization and economic freedom while employment rights are not robustly affected.

It is believed that based on the law of Comparative advantage, each region will now focus on areas or products they are best suited both naturally, physically, eonomically etc to produce/manufacture. It is assumed that free trade will enlarge the markets for domestic 
producers, allow them to benefit from the principle of economies of scale, make room for free competition and reward for hard work, provide opportunities and incentives for creativity as well as develop new technologies

One major setback in this principle, relative to developing countries and based on the law of Comparative advantages is that third world economies may end up only trading in primary products. No doubt, developed economies will have comparative edge in the production of secondary / intermediate goods. A typical example can be described with the Nigerian scenario which has low level of infrastructure, unstable interest rate and unstable prices of factors of production. This implies that planning and production of goods and services will attract higher costs than in the developed economy. So far, the only cheap factor of production in Nigeria is labour. The outcome is that highly priced industrial goods are exchanged for lowly priced primary goods/products. This postulation lends credence to the study of Bergh and Nilson (2010a) that freedom to trade internationally is robustly related to inequality and that social globalization along with deregulation is equally linked to inequality.

Baker and Nordin (2004) opined that dirty money binds the poor. They opine that the World Bank is narrowing its focus on corruption, rather than major problem of cross-border dirty money. This comprises of money that is illegally earned, illegally used or transferred and has three main facets namely: Criminal proceeds from trafficking and racketeering: commercial proceeds from trade and shady business transactions, often hidden in tax haven, and proceeds from greedy government officials. They revealed that about $\$ 50$ billion in aid goes to developing and transitional economies from richer nations. At the same time, roughly about $\$ 500$ billion in dirty money flows in the opposite direction out of poorer countries. In essence for every $\$ 1$ the World Bank distributes in assistance across the top of the table, $\$ 10$ is lost in illegal proceeds under the table. The above has a lot of implications for developing countries and may possibly account for the startling revelation discussed below.

Table 1: The Role of Developing Countries In Trade And Capital Flows.

\begin{tabular}{|l|l|l|l|}
\hline & $1980-82$ & $1987-90$ & $1996-97$ \\
\hline Exports (\%) & 32.7 & 27.2 & 34.0 \\
\hline Imports(Billions of \&) & 30.4 & 25.4 & 34.3 \\
\hline & 1856 & 2864 & 5459 \\
\hline Direct Investment(\%) & & & \\
\hline Portfolio Investment(\%) & 32.7 & 14.3 & 43.2 \\
\hline Total (Billion of \$) & 7.7 & 3.1 & 13.3 \\
\hline & 107 & 355 & 3119 \\
\hline
\end{tabular}

TABLE 2. Regional Differences Among Developing Countries In Trade \& Capital Flow

\begin{tabular}{|l|l|l|l|}
\hline Trade Flow & 1980 & 1990 & 1997 \\
\hline Asia & 28.3 & 51.4 & 52.3 \\
\hline Europe & 16.0 & 11.1 & 16.8 \\
\hline
\end{tabular}




\begin{tabular}{|l|l|l|l|}
\hline Latin America & 21.8 & 15.4 & 16.2 \\
\hline Middle East & 20.5 & 13.0 & 8.7 \\
\hline Africa & 13.3 & 8.7 & 6.1 \\
\hline Total & 100 & 100 & 100 \\
\hline & & & \\
\hline Capital Flows & 1980 & 1990 & 1997 \\
\hline Asia & 23.6 & 36.7 & 40.6 \\
\hline Europe & 16.1 & 13.3 & 17.5 \\
\hline Latin America & 36.1 & 21.6 & 34.4 \\
\hline Middle East & 10.3 & 10.2 & 2.0 \\
\hline Africa & 13.9 & 18.2 & 5.6 \\
\hline Total & 100 & 100 & 100 \\
\hline
\end{tabular}

Sources: IMF, Direction Trade Statistics Yearbook and World Bank Global Development Finance (1999)

From Table 1 above, we had nominal increase in trade in goods for the period of 1980 - 1997. The total value of goods also increased to 34 times during this period. However trade in non-goods witnessed sizeable increases during the same period. Shall we then say that globalization promotes trade in non-goods? However, from Table II, out of all the five regions highlighted: Latin America, Middle East and Africa witnessed reduction in the value of their trade flows. Infact, that of Middle East and Africa was highly significant, from 20.5 and 13.3 in 1980 to 8.7 and 6.1 in 1997. This glaring evidence suggests that globalization has only had negative effect on the flow of trade to these two regions. Similarly Capital flows for the three regions followed the same declining trend, from $10.3 ; 7.8$ and 13.9 respectively in 1980 to 2.0;4.3 and 5.6 in 1997.

One tends to as whether these countries do not have goods to trade with other countries? Even if that scenario is positive, what of Capital flows? Are the funds meant for certain economies only? So far both indices gave positive signal for Asia and Europe. No doubt, countries like China; India etc who were poor about 20 years ago have reaped a positive aspect of globalization which Table II above vividly explains. What trend does this portend?

The study explores further into the exchange rates of the countries and some poverty indices to analyze the situation. Table 3 below shows the exchange rates of some African countries between 1980 and 2002.

Table 3. Official Exchange rates and Black Market (Parallel Market) rates: Number of National Currency Units per US\$ in Africa Countries

\begin{tabular}{|l|l|ll|l|l|l|l|l|l|}
\hline S/N & & National Currency & 1980 & 1990 & 1995 & 1998 & 1999 & 2002 \\
\hline 1 & Country & Kwanza & 1 & - & 0.1 & $2,711.0$ & 392814.0 & $1,83830.0$ & 54.8 \\
& & & 2 & & 1,950 & 56574.0 & 118238.0 & $2,35000.0$ & 80.5 \\
\hline 2 & Angola & CFA Franc & 1 & 211.3 & 272.3 & 499.1 & 590.0 & 615.7 & 660.5 \\
& & & 2 & 209.5 & 2818 & 499.3 & 593.3 & 625.0 & 805.3 \\
\hline 3 & Benin Republic & CFA Franc & 1 & 0.8 & 1.9 & 2.8 & 4.2 & 3.9 & 5.8 \\
\hline
\end{tabular}




\section{MInstitute ${ }^{\text {Macrothink }}$}

Business and Economic Research ISSN 2162-4860

2014, Vol. 4, No. 1

\begin{tabular}{|c|c|c|c|c|c|c|c|c|c|}
\hline & & & 2 & 0.8 & 1.9 & 2.9 & 4.5 & 4.5 & 8.5 \\
\hline \multirow[t]{2}{*}{4} & \multirow[t]{2}{*}{ Botswana } & \multirow[t]{2}{*}{ Pula } & 1 & 211.3 & 2723 & 499.1 & 590.0 & 615.7 & 660.5 \\
\hline & & & 2 & 209.5 & 281.8 & 499.3 & 593.3 & 620.8 & 780.0 \\
\hline \multirow[t]{2}{*}{5} & \multirow[t]{2}{*}{ Burkina Faso } & \multirow[t]{2}{*}{ CFA Franc } & 1 & 90.0 & 171.3 & 249.8 & 447.8 & 563.6 & 1027.0 \\
\hline & & & 2 & 106.0 & 186.1 & 340.8 & 605.8 & 7150 & 950.0 \\
\hline \multirow[t]{2}{*}{6} & \multirow[t]{2}{*}{ Cameroom } & \multirow[t]{2}{*}{ Franc } & 1 & 209.2 & 70.0 & 518.5 & 602.1 & 588.4 & 660.5 \\
\hline & & & 2 & 209.5 & 00.7 & 499.3 & 605.8 & 625.0 & 805.0 \\
\hline \multirow[t]{2}{*}{7} & \multirow[t]{2}{*}{ Capeverde } & \multirow[t]{2}{*}{ CFA Franc } & 1 & Nill & Nill & 76.9 & 98.2 & 102.7 & 121.3 \\
\hline & & & 2 & 209.5 & 281.8 & 85.6 & 105.0 & 110.0 & 1325 \\
\hline \multirow[t]{2}{*}{8} & \multirow{2}{*}{$\begin{array}{ll}\text { Central } & \text { Africa } \\
\text { Republic } & \end{array}$} & \multirow[t]{2}{*}{ Escudo } & 1 & 40.2 & 272.3 & 499.1 & 590.0 & 615.7 & 660.5 \\
\hline & & & 2 & 211.3 & 281.8 & 499.3 & 605.8 & 625.0 & 805.0 \\
\hline \multirow[t]{2}{*}{9} & \multirow[t]{2}{*}{ Chad } & \multirow[t]{2}{*}{ CFA Franc } & 1 & 209.5 & 272.3 & 374.4 & 590.0 & 615.7 & 660.5 \\
\hline & & & 2 & 211.3 & 28.18 & 390.6 & 605.8 & 625.7 & 805.0 \\
\hline \multirow[t]{2}{*}{10} & \multirow[t]{2}{*}{ Comoros Republic } & \multirow[t]{2}{*}{ CFA Franc } & 1 & 209.5 & 0.1 & 6254.0 & 422.5 & 461.8 & 495.4 \\
\hline & & & 2 & 211.3 & 738.1 & 7452.0 & 475.0 & 505.0 & 610.0 \\
\hline \multirow[t]{2}{*}{11} & Democratic & \multirow[t]{2}{*}{ CFA Franc } & 1 & 209.5 & 272.3 & 499.1 & $129,101.0$ & $140,286.0$ & 409.4 \\
\hline & Republic of & & 2 & 0.1 & 281.8 & 409.3 & 160500.0 & $170,550.0$ & 805.0 \\
\hline 12 & Congo Republic & Congolese & 1 & 6.4 & 2723 & 499.1 & 605.8 & 625.7 & 660.5 \\
\hline & (Brazzaville) & & 2 & 211.3 & & & 608.8 & 625.7 & 680.00 \\
\hline & & & & 209.5 & & & & & \\
\hline 13 & Coted'Ivoire & CFA Franc & 1 & 211.3 & 281.8 & 499.3 & 590.0 & 61.7 & 660.5 \\
\hline & (Ivory Coast) & & 2 & 209.5 & & & 605.8 & 625.7 & 805.0 \\
\hline 14 & Djibouti & Djibouti & Franc & 1980 & 1990 & 177.7 & 177.7 & 177.7 & 175.0 \\
\hline & & 1 & & 177.7 & 177.7 & 198.5 & 183.9 & 183.9 & 195.0 \\
\hline & & & 2 & & & & & & \\
\hline 15 & Equatorial Guinea & CFA Franc & 1 & Nill & 191.8 & 499.1 & 590.0 & 615.7 & 660.5 \\
\hline & (Fernando Island) & & 2 & 110.6 & 272.3 & 586.4 & 593.3 & 625.7 & 805.0 \\
\hline & & & & 209.5 & & 68 & & & \\
\hline & & & & & & 10.5 & & & \\
\hline 16 & Eritrea & Birr & 1 & - & 281.8 & 5.9 & 7.4 & 625.7 & 8.5 \\
\hline & & & 2 & - & - & 10.4 & 10.8 & 8.5 & 11.9 \\
\hline & & & & & & 499.1 & & & \\
\hline 17 & Ethiopia & Birr & 1 & 211.3 & - & 499.3 & 6.9 & 11.5 & 12.7 \\
\hline & & & 2 & 209.5 & 2.6 & 9.5 & 11.8 & 7.5 & 66.05 \\
\hline & & & & & & 10.4 & & & \\
\hline 18 & Gabon & CFA Franc & 1 & 211.3 & 60 & $1,200.4$ & 590.0 & 12.5 & 805.0 \\
\hline & & & 2 & 209.5 & 272.3 & 991.4 & 593.3 & 615.7 & 23.0 \\
\hline 19 & Gambia & Dalasi & 1 & 1.7 & 281.8 & 499.1 & 10.6 & 625.7 & 30.6 \\
\hline & & & 2 & 1.7 & 7.9 & 499.3 & 11.0 & 11.4 & $8,231.4$ \\
\hline 20 & Ghana & Code & 1 & 9.6 & 8.3 & 9.5 & $2,314.1$ & 12.5 & $9,100.1$ \\
\hline & & & 2 & 15.9 & 326.3 & 10.4 & $2,500.0$ & $2,647.3$ & 971.0 \\
\hline 21 & Guinea & Franc & 1 & 19.0 & 360.8 & $1,200.4$ & $1,236.8$ & $2,700.0$ & $1,850.0$ \\
\hline
\end{tabular}


MI Macrothink

Business and Economic Research ISSN 2162-4860

2014, Vol. 4, No. 1

\begin{tabular}{|c|c|c|c|c|c|c|c|c|c|}
\hline & & & 2 & 41.7 & 660.2 & $1,224.3$ & $1,350.0$ & $1,343.6$ & 660.5 \\
\hline \multirow[t]{2}{*}{22} & Guinea Bissau & CFA Franc & 1 & 0.8 & 693.3 & 991.4 & 590.0 & $1,610.0$ & $28,500.0$ \\
\hline & & & 2 & Nill & 33.6 & $1,017.9$ & $26,890.0$ & 615.7 & 79.9 \\
\hline \multirow[t]{2}{*}{23} & Kenya & Shilling & 1 & 7.4 & Nill & 278.0 & 60.4 & $27,850.0$ & 85.0 \\
\hline & & & 2 & 8.2 & 22.9 & $19,167.0$ & 650 & 704 & 93 \\
\hline \multirow[t]{2}{*}{24} & Lesotho & Maloti & 1 & 0.8 & 23.3 & 51.4 & 5.5 & 75.0 & 10. \\
\hline & & & 2 & nill & 2.6 & 53.4 & 5.8 & 6.1 & 60.2 \\
\hline \multirow[t]{4}{*}{25} & Liberia & Dollar & 1 & 1.0 & 2.7 & 3.6 & 41.5 & 6.1 & 66.8 \\
\hline & & & 2 & 1.0 & 1.0 & 3.7 & 48.5 & & $7,500.0$ \\
\hline & & & & & & 1.0 & & & \\
\hline & & & & & & 42.3 & & & \\
\hline \multirow[t]{3}{*}{26} & Madagascar & Malagasy Franc & 1 & 211.3 & $1,494.2$ & $4,265.6$ & $5,441.4$ & 6.5 & $7,800.0$ \\
\hline & & & 2 & 265.0 & $1,589.2$ & 4387.5 & $5,582.1$ & 41.9 & \\
\hline & & & & & & & & 51.0 & \\
\hline \multirow[t]{2}{*}{27} & Malawi & Kwacha & 1 & 0.8 & 2.7 & 15.3 & 31.1 & $6,283.8$ & 81.6 \\
\hline & & & 2 & 1.6 & 3.3 & 16.7 & 35.5 & $6,450.0$ & 92.0 \\
\hline \multirow[t]{2}{*}{28} & Mail & CAF Franc & 1 & 211.3 & 272.3 & 499.1 & 590.0 & 44.1 & 660.5 \\
\hline & & & 2 & 209.5 & 281.8 & 499.3 & 605.8 & 52.0 & 805.0 \\
\hline \multirow[t]{3}{*}{29} & Mauritania & Onguiya & 1 & 45.9 & 80.6 & 17.4 & 189.0 & 625.7 & 267.0 \\
\hline & & & 2 & 65.0 & 90.0 & 18.2 & 197.5 & 209.2 & 268.5 \\
\hline & & & & & & & 24.0 & & \\
\hline \multirow[t]{2}{*}{30} & Mauritius & Rupee & 1 & 7.7 & 14.9 & $8,889.8$ & 25.4 & 220.0 & 29.5 \\
\hline & & & 2 & - & 15.7 & $9,865.7$ & $11,874.6$ & 25.0 & 30.0 \\
\hline \multirow[t]{2}{*}{31} & Mozambique & Metical & 1 & 32.4 & 929.1 & 3.6 & 12750.0 & 27.0 & $23,346.5$ \\
\hline & & & 2 & 80.0 & 1360.5 & 4.1 & 55 & & $25,840.0$ \\
\hline \multirow[t]{2}{*}{32} & Namibia & Rand & 1 & 0.8 & 2.6 & 499.1 & 6.0 & $12,775.1$ & 8.1 \\
\hline & & & 2 & 0.9 & 3.0 & 499.3 & 590.0 & 13550.0 & 9.2 \\
\hline \multirow[t]{2}{*}{33} & Niger & CFA Franc & 1 & 211.3 & 272.3 & 21.9 & 593.3 & 615.7 & 660.5 \\
\hline & & & 2 & 209.5 & 281.8 & 78.3 & 21.9 & 620.8 & 780.0 \\
\hline \multirow[t]{2}{*}{34} & Nigeria & Naira & 1 & 0.8 & 9.2 & 262.2 & 85.0 & 92.3 & 128.8 \\
\hline & & & 2 & 0.9 & 9.3 & 2681 & 312.3 & 101.5 & 140.0 \\
\hline \multirow[t]{2}{*}{35} & Rwanda & Franc & 1 & 211.3 & 82.6 & $1,420.3$ & 360.0 & 333.9 & 485.0 \\
\hline & & & 2 & 209.5 & 104.2 & $1,503.3$ & $6,883.2$ & 350.0 & 575.0 \\
\hline \multirow[t]{2}{*}{36} & Sao Tome and & Bora & 1 & 0.8 & 143.3 & 499.1 & $7,100.0$ & $7,119.0$ & $7,250.0$ \\
\hline & Principle & & 2 & 0.9 & Nill & 499.3 & 590.0 & $7,200.0$ & $7,300.0$ \\
\hline \multirow[t]{3}{*}{37} & Senegal & CFA Franc & 1 & 92.8 & 272.3 & 4.8 & 593.3 & 615.7 & 660.5 \\
\hline & & & 2 & 115.0 & 281.8 & 5.5 & 5.3 & 625.0 & 805.0 \\
\hline & & & & 34.8 & & & & & \\
\hline \multirow[t]{3}{*}{38} & Seychelles & Rupee & 1 & Nill & 5.3 & 755.2 & 5.4 & 5.3 & 5.6 \\
\hline & & & 2 & 211.3 & 5.9 & & $1,563.6$ & 5.5 & \\
\hline & & & & 209.5 & & & & & \\
\hline \multirow[t]{2}{*}{39} & Sierra Leone & Leone & 1 & 6.4 & 151.4 & 741.3 & 1.605 .0 & $1,804.2$ & 6.6 \\
\hline & & & 2 & 6.5 & 470.6 & & $5,919.3$ & $1,950.0$ & \\
\hline
\end{tabular}




\section{MlMacrothink}

Business and Economic Research ISSN 2162-4860 2014, Vol. 4, No. 1

\begin{tabular}{|c|c|c|c|c|c|c|c|c|c|}
\hline & & & & $\begin{array}{l}1.0 \\
1.4\end{array}$ & & & & & \\
\hline \multirow[t]{2}{*}{40} & \multirow[t]{2}{*}{ Somalia } & \multirow[t]{2}{*}{ Shilling } & 1 & 6.3 & $1,896.1$ & $1,949.2$ & 5.5 & $2,200.0$ & $1,700.0$ \\
\hline & & & 2 & 9.9 & $1,982.1$ & $6,549.2$ & 6.0 & $6,155.0$ & $2,120.0$ \\
\hline \multirow{2}{*}{41} & \multirow{2}{*}{ South Africa } & \multirow{2}{*}{ Rand } & 1 & 28.8 & 2.6 & 3.6 & 2.008 .0 & 6.1 & $2,620.0$ \\
\hline & & & 2 & 0.9 & 2.7 & 3.7 & 250.0 & 7.4 & $7,250.0$ \\
\hline \multirow[t]{2}{*}{42} & \multirow[t]{2}{*}{ Sudan } & \multirow[t]{2}{*}{ Dinar } & 1 & 0.6 & 12.2 & 580.9 & 5.5 & 2525.5 & 9.3 \\
\hline & & & 2 & 1.0 & 23.6 & 77.5 & 6.0 & & 9.8 \\
\hline \multirow[t]{2}{*}{43} & \multirow[t]{2}{*}{ Swaziland } & \multirow[t]{2}{*}{ Lilangeni } & 1 & 0.8 & 2.6 & 3.6 & 664.7 & 6.1 & $2,587.0$ \\
\hline & & & 2 & & 2.7 & 3.9 & 730.0 & 7.4 & $3,750.0$ \\
\hline \multirow[t]{2}{*}{44} & \multirow[t]{2}{*}{ Tanzania } & \multirow[t]{2}{*}{ Shilling } & 1 & 0.9 & 195.1 & 574.8 & 590.0 & 744.8 & 9.6 \\
\hline & & & 2 & & 292.4 & 587.3 & 593.3 & 950.8 & 10.8 \\
\hline \multirow[t]{2}{*}{45} & \multirow[t]{2}{*}{ Togo } & \multirow[t]{2}{*}{ CFA Franc } & 1 & 8.2 & 272.3 & 499.1 & 590.0 & 615.7 & 985.0 \\
\hline & & & 2 & 21.0 & 291.8 & 499.3 & 593.3 & 620.8 & $1,100.0$ \\
\hline \multirow[t]{2}{*}{46} & \multirow[t]{2}{*}{ Uganda } & \multirow[t]{2}{*}{ Shilling } & 1 & 1.0 & 319.6 & 932.5 & $1,149.7$ & $1,362.1$ & $1,843.0$ \\
\hline & & & 2 & 75.7 & 685.8 & $1,076.2$ & $1,305.0$ & $1,710.0$ & $1,920.0$ \\
\hline \multirow[t]{3}{*}{47} & \multirow[t]{3}{*}{ Zimbabwe } & \multirow[t]{3}{*}{ Dollar } & 1 & 0.6 & 2.4 & 8.7 & 21.4 & 38.3 & 55.1 \\
\hline & & & 2 & 1.9 & 4.5 & 9.2 & 39.2 & 57.5 & 60.9 \\
\hline & & & & & & & 58.7 & 66.6 & 79.9 \\
\hline \multirow[t]{2}{*}{48} & \multirow[t]{2}{*}{ Algeria } & \multirow[t]{2}{*}{ Dinar } & 1 & 3.8 & 9.0 & 47.7 & 58.7 & 138.0 & 150.0 \\
\hline & & & 2 & 10.9 & 29.8 & 131.9 & 129.8 & 3.4 & 4.8 \\
\hline \multirow[t]{2}{*}{49} & \multirow[t]{2}{*}{ Egypt } & \multirow[t]{2}{*}{ Pound } & 1 & 0.7 & 2.2 & 3.4 & 3.4 & 3.7 & 5.9 \\
\hline & & & 2 & 0.8 & 2.6 & 3.4 & 34 & & \\
\hline 50 & Libya & Dinar & 1 & 0.3 & 0.3 & 0.3 & 0.6 & 0.8 & 1.2 \\
\hline & & & 2 & 0.5 & 1.0 & 1.3 & 2.2 & 2.4 & 2.6 \\
\hline 51 & Morocco & Dinar & 1 & 3.9 & 8.2 & 8.5 & 9.6 & 9.8 & 10.6 \\
\hline & & & 2 & 4.1 & 9.3 & 8.6 & 11.1 & 11.2 & 11.5 \\
\hline 52 & Tunisia & Dinar & 1 & 0.4 & 0.9 & 0.9 & 1.1 & 1.2 & 1.4 \\
\hline & & & 2 & 0.4 & 0.9 & 0.9 & 1.1 & 1.4 & 1.6 \\
\hline
\end{tabular}

Notes: 1 after the currency means the Official exchange rate while 2. denotes Black market (parallel) rate.

Sources: I.Africa Development indicators 2003: The World Bank Washington, D.C. 2003

II. West Africa International Magazine, London, 13-19 and

III. World Almanac and Book of Facts 2003, New York USA, 2003, pages 756-855.

From Table III above only South Africa's exchange rate improved from Rand 28.8 to $\$$ in 1980 to Rand 9.3to \$1 in 2002. Nigeria naira moved from $\$$. 8 to $\$ 1$ in 1980 to $\$ 128.8$ to $\$ 1$ in 2002 . The scenario is pathetic because prices of goods have increased, yet they have a poorly valued currency to pay from them. As earlier stated, the paper examines some poverty indices such as gross national product and gross domestic product for the countries covered in Table 3 above. This is to examine the level of correlation in the data and whether it actually tells a story. 
Table 4. Per Capital National Product of Africa Countries, in Selected Years, 1980-2002 in US\$

\begin{tabular}{|c|c|c|c|c|c|c|c|}
\hline $\mathrm{S} / \mathrm{N}$ & Country & 1980 & 1990 & 1992 & 1995 & 2002 & 2002 as \% of 1980 \\
\hline 1 & Angola & 1,350 & 840 & 960 & 940 & 1,100 & 81.48 \\
\hline 2 & Benin Republic & 1,110 & 1,360 & 1,370 & 1,350 & 1,030 & 92,7 \\
\hline 3 & Botswana & 2,505 & 4,730 & 483 & 5360 & 6,600 & 263.4 \\
\hline 4 & Burkina Faso & 960 & 990 & 990 & 920 & 1,000 & 92.3 \\
\hline 5 & Burundi & 1,120 & 920 & 910 & 750 & 720 & 54.5 \\
\hline 6 & Cameroon & 1,850 & 1,970 & 1,920 & 1,1660 & 1,700 & 92.3 \\
\hline 7 & Cape Verde & 1,620 & 1,980 & 1,900 & 1,810 & 1,700 & 1118.8 \\
\hline 8 & Central Africa Republic & 1,840 & 1,470 & 1,460 & 1,350 & 1,700 & 82.3 \\
\hline 9 & Chad & 1,140 & 1,280 & 1,250 & 1,210 & 1000 & 875 \\
\hline 10 & Comoros Republic & 760 & 540 & 620 & 640 & 720 & 94.6 \\
\hline 11 & $\begin{array}{l}\text { Democratic Republic of } \\
\text { Congo }\end{array}$ & 670 & 620 & 600 & 575 & 600 & 89.7 \\
\hline 12 & $\begin{array}{ll}\text { Congo } & \text { Republic } \\
\text { (Brazzaville) } & \\
\end{array}$ & 1,600 & 980 & 920 & 910 & 1,000 & 62.5 \\
\hline 13 & Cote d' Ivoire (Ivory Cost) & 2,540 & 2,800 & 2,800 & 1,670 & 1,600 & 62.3 \\
\hline 14 & Djibouti & 1,054 & 1,050 & 910 & 850 & 574 & 54.5 \\
\hline 15 & $\begin{array}{l}\text { Equatorial Guinea } \\
\text { (Fernando Island) }\end{array}$ & 2,860 & 2,350 & 1980 & 2,100 & 2,000 & 70.9 \\
\hline 16 & Eritrea & 2,100 & 1,110 & 1,110 & 1,110 & 600 & 28.6 \\
\hline 17 & Ethiopia & 2,100 & 1,110 & 5,050 & 5,8506 & 6,300 & 69.5 \\
\hline 18 & Gabon & 9,050 & 5,050 & 1,350 & 1,350 & 1,110 & 86.8 \\
\hline 19 & Gambia & 1,280 & 1,350 & 1,430 & 1,370 & 1,900 & 90.7 \\
\hline 20 & Ghana & 2,090 & 1,430 & 1,520 & 1,560 & 1,300 & 68.0 \\
\hline 21 & Guinea & 1,910 & 1,520 & 1,240 & 1,220 & 850 & 106.7 \\
\hline 22 & Guinea Bissau & 802 & 1,240 & 1,330 & 1,260 & 1500 & 80.0 \\
\hline 23 & Kenya & 1,875 & 1,330 & 1,650 & 1,690 & 2,400 & 112.2 \\
\hline 24 & Lesotho & 2,140 & 1,650 & 1,310 & 1,270 & 1,100 & 56.2 \\
\hline 25 & liberia & 1,90 & 1,310 & 1,230 & 740 & 800 & 55.6 \\
\hline 26 & Madagascar & 1,440 & 1,230 & 820 & 860 & 900 & 94.7 \\
\hline 27 & Malawi & 950 & 820 & 920 & 850 & 850 & 88.9 \\
\hline 28 & Mauritania & 910 & 920 & 2,540 & 2,450 & 2,000 & 84.8 \\
\hline 29 & Mauritus & 2,355 & 2,540 & 8,960 & 8,420 & 10,400 & 270.2 \\
\hline 30 & Mozambique & 3,850 & 8,960 & 1,140 & 1,140 & 1,000 & 46.8 \\
\hline 31 & Namibia & 2,140 & 1,140 & 4030 & 4,160 & 4300 & 145.4 \\
\hline 32 & Niger & 2,960 & 4030 & 1,290 & 1,190 & 1,090 & 43.2 \\
\hline 33 & Nigeria & 2,100 & 1,290 & 1,310 & 1,190 & 900 & 100 \\
\hline 34 & Rwanda & 2,400 & 1,290 & 1,390 & 1,340 & 1,100 & 42.9 \\
\hline 35 & Sao Tome and Principle & 900 & 1,310 & 1,310 & 1,190 & 900 & 100 \\
\hline 36 & Senegal & 2,560 & 1,390 & 1,390 & 1,340 & 1,100 & 42.9 \\
\hline 37 & Seychelles & 1,660 & 1,780 & 1,780 & 1,550 & 1,600 & 96.2 \\
\hline
\end{tabular}




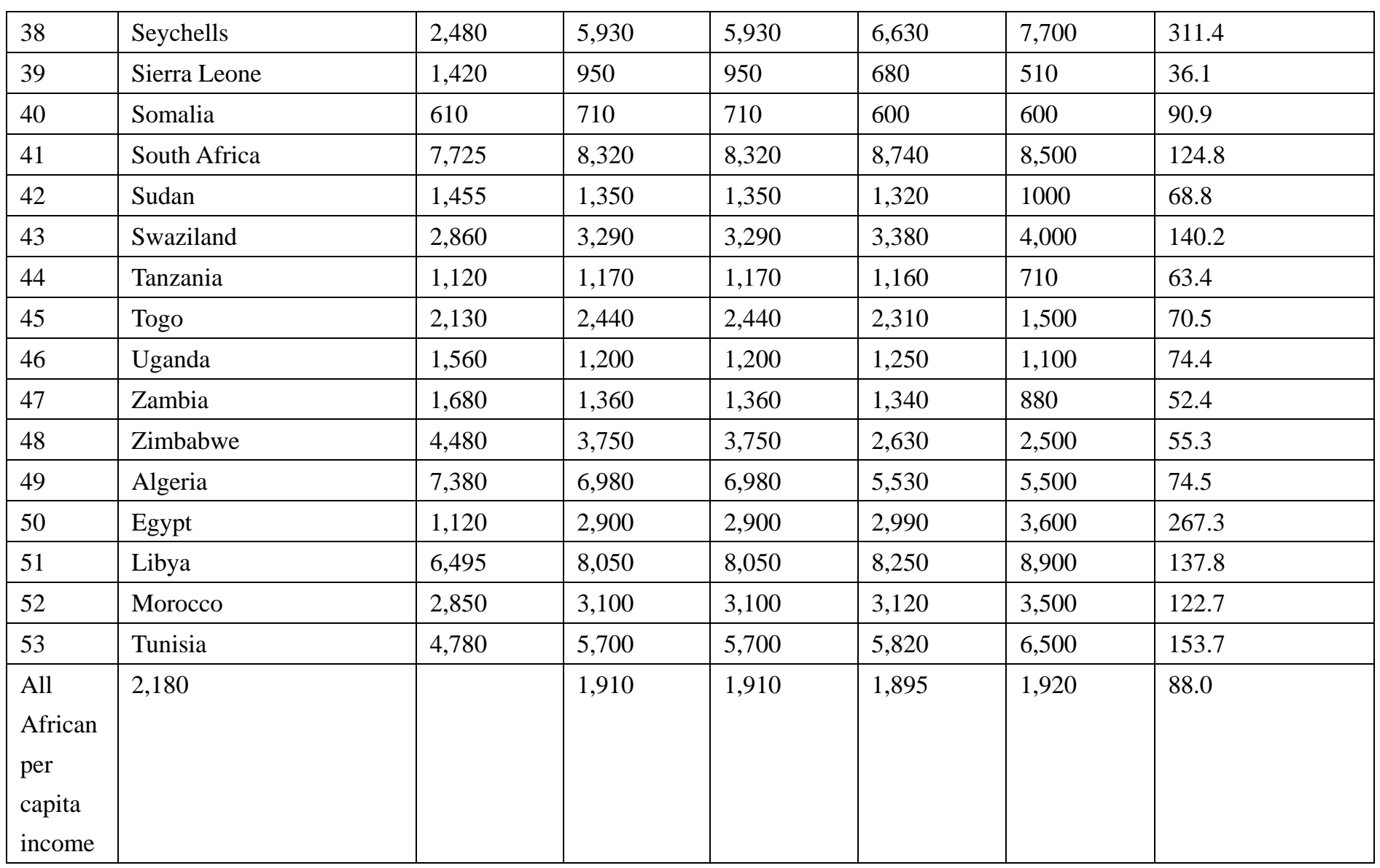

The Per capital Gross National Product of these Africa countries in Table IV over the same period (22 years) did not show any encouraging signal. This suggests that little or no improvement can be expected with the living standard of the populace over this period. The outcome of the rate of development cannot be compared to that of the developed countries as detailed in Table V below.

Table 5. Per Capita Gross Domestic Product In Selected Major Creditor Countries (Paris Club And Other), 1980-2002 In Us\$

\begin{tabular}{|l|l|l|l|l|}
\hline S/N & Country & 1980 & 2002 & 2002 AS \% OF 1980 \\
\hline 1 & Australia & 11550 & 23,200 & $200.5 \%$ \\
\hline 2 & Austria & 14,500 & 25,000 & $172.4 \%$ \\
\hline 3 & Belgium & 13,950 & 25,300 & $181 . \%$ \\
\hline 4 & Canada & 15,950 & 24,800 & $155.5 \%$ \\
\hline 5 & Demark & 16,850 & 25,500 & $151.4 \%$ \\
\hline 6 & Finland & 13,650 & 22,900 & $135.9 \%$ \\
\hline 7 & France & 19.750 & 24,400 & $174.4 \%$ \\
\hline 8 & Germany & 10,720 & 23,400 & $118.5 \%$ \\
\hline 9 & Greece & 5,180 & 17,200 & $160 \%$ \\
\hline 10 & Ireland & 6,850 & 21,600 & $417.0 \%$ \\
\hline 11 & Israel & 13,050 & 18,900 & $275 . \%$ \\
\hline 12 & Italy & 18,450 & 22,100 & $169.4 \%$ \\
\hline
\end{tabular}




\section{MInstitute ${ }_{\text {Mnim }}^{\text {Macrothin }}$}

Business and Economic Research

ISSN 2162-4860

2014, Vol. 4, No. 1

\begin{tabular}{|l|l|l|l|l|}
\hline 13 & Japan & 17,040 & 24,900 & $135.0 \%$ \\
\hline 14 & Netherlands (Holland) & 12,650 & 24,400 & $143 \%$ \\
\hline 15 & New Zealand & 16,850 & 17,700 & $139.9 \%$ \\
\hline 16 & Norway & 5,350 & 27,700 & $164.4 \%$ \\
\hline 17 & Portugal & 6,850 & 15,800 & $295.3 \%$ \\
\hline 18 & Spain & 18,450 & 18,000 & $262.8 \%$ \\
\hline 19 & Sweden & 20,550 & 22,200 & $120.3 \%$ \\
\hline 20 & Switzerland & 12,550 & 28,600 & $139.2 \%$ \\
\hline 21 & United Kingdom & 14,061 & 22,800 & $181.2 \%$ \\
\hline 22 & United State of Africa & & 36,200 & $245.4 \%$ \\
\hline & All 22 countries & & 27,000 & $192 . \%$ \\
\hline
\end{tabular}

Sources: World Development Indicators 2003, Table 1.1 Pages 12-4: World Almanac and Book of facts 2003, New York, 2003, pages 756-855

A cursory look at the table reveals that the rate of development during the same period for the developed economies was significant. Of interest was that of Spain, Ireland, Israel, Portugal, United states of America with 262.8\%, 417.\% 275.9\%,295.3\%,200.5\%, 181.2\% and 245.4\% respectively. Most African countries rate ranged from 28.6 to $100 \%$

Apart from the above analogy, several anti-globalization crusaders suggest that globalization is a form of window dressing by the imperialist powers to dominate the world through their economic might and terms of trade. The avenue for free trade and competition has only favoured a few giant corporations who now dominate the globe and currently possess more power than an elected government in some countries. According to Sewell (1992), these Multinationals use their economic muscle to crush all opposition and buy politician of all colours and also engage in corruption as a means of protecting their system. These global companies have no other interest apart from conquering new areas of the world and making astronomical profits. According to Guillen (2001) proponents and enemies of globalization alike suggest the demise of the nation state which has become irrelevant and helpless to deal with rootless International Capital. However Wade (1996) sees globalization as a feeble process but has not challenged the nation state and other fundamental features of the modern world.

\section{b) Information Technology (IT)}

The World has more or less become a global village through linkages afforded by Computerization and the Web. This to a large extent facilities trade in Goods, Services, Capital flows etc. As a result of this processes, transaction and search cots are reduced.

A perusal of the arguments of the opponents of globalization reveals that to a large extent that the Internet plays a major role in the success of their protest and demonstrations. They identify and publicize targets, solicit and encourage support organize and communicate information, recruit, raise funds and plan using the internet and cell phones.

Similarly, the Multinationals have maximized the advantages accruing from Information Technology. At little or no cost, they shop around for where to locate their business. To a large 
extent, developing economies have received four considerations, but not without its lamentations. Places like China where they are happy to work a whole 14 hours day's job for as little as 50p have received fair consideration. In essence, they search for cheap wage locations, yet they don't want to pay for it.

This situation has led to accusation of abuse of corporate power by protesters. They claim that their activities are tantamount to Social Injustice, Unfair labour practices, poor working and living conditions, lack of concern for the environment, mismanagement of natural resources and ecological damage. The third World is not only treated as an area for exploitation, but for dumping and experimentation. The Miami herald was stated to have reported the death of a 15 year old farm worker due to poison by a highly toxic America Cyanamid product. These are legal Pesticides imported into the country only to account for death toll of about 220,000 a year by the World Health Organization and 25Million incidents of illness including Chronic neurological damage

This situation can best be likened to that in which the Multinationals reap super-profits worldwide while their workers and the environment are subjected to super exploitation and killings. According to Sewell (2001), Ezequiel Tinajero, was 16 years old when he went to work at the Auto Trim plant in Matamoros on Mexico's northern border with United States of America. For 12 hours a day, Monday to Saturday, he glued leather trim to the steering wheels for luxury cars. For most of that time till he reached his 20's, he was suffering from chronic nausea, headaches and breathing problems, and his hair began to fall out. In 1995, his wife gave birth to a daughter with anencephaly, a rare condition in which the Child has no brain. The baby died two hours after birth. How many Tinajero's have lost their lives in the hands of these giant corporations?

While it is often argued that the Institutions responsible for globalization notably World Bank, IMF and WTO, are equally guilty because they are mere servants of the Corporate giants, this can be viewed differently. This is because this paper has highlighted the gains and pains of globalization; it has effect on the developing countries and even the opposers of globalization through the magnitude of gain or pain being witnessed. In view of this, this paper submits that the corporate giants merely accepted the concepts of globalization from the proposing institutions after they observed the inherent loopholes which they have tapped to their maximum advantage and disadvantages of the developing countries.

\section{Inferences}

$>$ A large number of the world populace estimated at about 4billion people have suddenly entered the world economy through access to internet and the web.

$>$ Sequel to the WTO negotiations in Seattle, old barriers are now coming down, resulting in transfer of Technology and Innovations to developing countries

$>$ Even with globalization, a proportion of countries in the world estimated at about $25 \%$ are suffering from increased poverty. This is expected to reduce if inherent loopholes in the processes are dealt with. 


\section{$\triangle$ Macrothink}

Business and Economic Research

ISSN 2162-4860

2014, Vol. 4, No. 1

> Giant corporations' call for competitiveness is a disguised monopoly as they are mere self serving organizations that violate human rights, environmental degradation and pollution in search of cheap labour

$>$ The squeeze by multinationals grinds the working masses to deeper poverty and squalor including child labour

$>$ As long as multinationals' rule the earth, many will continue to experience deeper poverty amidst world's plenty in the hands of the minority

$>$ Capital flows between advanced countries and developing nations do not get reasonable share.

$>$ Gap between the rich and poor nations widened

$>$ Developing counties that are primary producers with poor infrastructural developing count their losses with globalization. Consider the under listed staggering figures since the emergence of globalization according to the United Nations Annual Development report.

$>54$ Countries saw a decline of average incomes throughout the 1990's

$>21$ Countries deteriorated in terms of human Capital development as measured by Income, Life expectancy and Literacy

$>$ 30,000 Children die daily from illnesses that are totally preventable

$>$ 500,000 Women die yearly during Pregnancy or Children birth number of people killed in armed conflicts since World War II

$>$ In Zimbabwe, the average life expectancy declined from 56 in 1970's to 33 in 1990's while that of UK rose from 72 to 78 during the same period.

$>$ In Sierras Leone, 363 Children out of every 1000 do not spend up to 5 years before they die, just as only 4 out of every 1000 in Norway do not survive

\section{Recommendation/Conclusion}

This paper has highlighted both the advantages and disadvantages of globalization. It has also become crystal clear that countries that are majorly primary producers only exchange their goods for highly priced products by the developed countries. No doubt, countries like China, India etc have positive result from globalization because they are not majorly primary producers. It therefore means that other developing countries should do internal integration. The major concept of economies of scale or law of comparative advantage upon which globalization is hinged upon may not work positively for the developing countries. The sincerity relative to pricing by the developed countries has totally failed. Developing countries must learn how to turn their raw materials to semi- products or finished products of very good standard so that they can compete effectively with other countries. If China and India can change the fortunes of their economy, other developing countries can. This has to be handled with care due to possible labour market induced effects. According to Hillman (2008) there could be significant consequences for perceived social justice and efficiency emanating from globalization induced labour market effects. Likewise there is a possibility of negative impact of democracy on ethnic conflict as a result of globalization more so that robust evuidence was found in the developing countries (Bezemer \& Jong-A-Pin; 2013).

To achieve the above goal, the level of infrastructural facilities must be improved, so also 
communication and literacy levels. This will assist the populace to maximize the gains of globalization. They will not make do with what get, rather search globally for the best place to effect their transaction. Of importance is the political upheaval pervading the developing nations. Where there is no stability, not only is planning difficult, investors will be scared of coming to that environment. I am sure that has largely accounted for the poor consideration they get from the giant corporations. Closely related are some economies that are still being ruled by military or politicians that perpetuate themselves in office. Developing countries should desist from actions that draw them backwards.

It is easy to destroy than to build. The WTO negotiations in Seattle for removal of trade barriers currently may not work for all economies. Certain countries still need to protect their infant industries so that the elephant corporations do not scuffle them to death. I am sure a mother will never throw the same challenges before a 3month old baby and a ten year old child. Eliminating trade barriers totally and opening borders for free movement of goods and services is a policy that developing countries need to view technically and with caution

The G8 summit held in June 2004 considered reducing remittances by the migrants which had risen to $\$ 75$ billion, far in excess of the \$52billion of Oversea Development Assistance (ODA). This fund should be monitored to ensure proper targeting for those in dire need. The study by Bjornskov (2010) suggests that aid to democratic countries may benefit only the political elite which may result in ineffective funding for the desired purpose. In the same vein, developing economies should formulate policies that will:

$\checkmark$ Stipulate minimum amount payable to each worker

$\checkmark$ Adopt profit sharing formula for staff mostly in giant corporations

$\checkmark$ Peg amount to be remitted back by these corporation while the remaining should be ploughed back to the economy where the money was generated from or where the corporation is located

$\checkmark$ Engage employers in welfare packages that will assist the workers in case of any fallout.

Lastly, the globalization institutions should go back to the drawing board and examine majorly the inherent loopholes currently being utilized with a view to addressing them. It is possible to find robust and positive effect of economic globalization on life expectancy as postulated by Bergh \& Nilson (2010b); only if the loopholes are properly addressed. The current trend of the rich becoming richer and while the poor becoming poorer is a major setback to globalization. If it continues, it will generate outcries more than the present opponents. Remember, a hungry man is an angry man. To have just about 500 companies ruling the world is absurd. Let us change our orientation to how we can improve the lots of the fellow human beings than see profit making as a religion that has to be pursued.

Having stated the aforementioned, the study opines that globalization to the developing economies so far has been a pain, but can be harnessed to be a gain just like China and India. The world is large enough and the resources abundant for a better living standard by all the occupants. 


\section{References}

Aluko Sam (2003). Background and Africa's Economic development, Selected Papers for the 2003 Annual Conference - The Nigeria Economic Society.

Avi-Yonah, R. (2000). Globalization, Tax Competition and the Fiscal Crisis of the Welfare State. Harvard Law Review, 13, 1573. http://dx.doi.org/10.2307/1342445

Avi-Yonah, R. (2003). Globalization and Tax Competition: Implication for Developing Countries. being

Baker R. \& Nodin, J. (2004). How Dirty Money Binds The Poor. Financial Times, $13^{\text {th }}$ October.

BBC News (2001). What is Anti-Globalization. U.K, May.

Bergh, Andreas \& Nilsson, Therese, (2010a). Do liberalization and globalization increase income inequality?, European Journal of Political Economy, Elsevier, vol. 26(4), 488-505. http://dx.doi.org/10.1016/j.ejpoleco.2010.03.002

Bergh, Andreas \& Nilsson, Therese, (2010b). Good for Living? On the Relationship between Globalization and Life Expectancy World Development, Elsevier, vol. 38(9), 1191-1203.

Bezemer, D., \& Jong-A-Pin, R. (2013). Democracy, Globalization and Ethnic Violence; $\begin{array}{llll}\text { Journal of Comparative } \quad \text { Economics, } & 41(1), & 108-125 .\end{array}$ http://dx.doi.org/10.1016/j.jce.2012.06.003

Bjornskov, C (2010). On globalization and human rights: the importance of types of globalization-University of Aarhus Working Paper

Conference Paper presented to the $11^{\text {th }}$ Meeting of United Nations Ad Hoc Group of Experts on International Cooperation in Tax Matters, Geneva, 15-19, December

CSIS (2000). Anti-globalization-A Spreading Phenonmenon. Canadian Security Intelligence Service, August.

De Soysa, I, \& Vadlamannati, K (2011). Does being bound together suffocate or liberate? The effects of economic, social and political globalization on Human Rights 1981-2005; Kyklos 64(1), 20-53. http://dx.doi.org/10.1111/j.1467-6435.2010.00493.x

Dreher, A.; Gassebner, M., \& Siemers, L. (2010). Globalization, Economic Freedom and Human Rights; CEGE Discussion Papers, No 115

Guillen Mauro F (2001). Is Globalization Civilizing, Destructive or Feeble? A critique of five keys debates in the Social Science Literature. October

Haus de Vreiji (2001). Dialogue with Anti-Globalization Activists. Radio Nether, Tuesday 21 August

Hillman, A (2008). Globalization and Social Justice, Singapore Economic Review, 53(2), 173-189. http://dx.doi.org/10.1142/S0217590808002896 
Mitchell David (2003). Growing World Poverty and Conflict shows the Barbarity of Capitalism, July

Potrafke, N. (2013). Globalization and Labour Market Institutions: International Empirical Evidence; IFO Working Paper, No 154.

Prasad, E; Kose, M; Rogoff K., \& Wei, S (2003). Effects of Financial Globalization on Developing Countries: Some Empirical Evidence. $I M F$

Roseanna, M. (1997). Modernity and Globalization.

Sewell Rob (2001). Imperialism, Globalization and the Way Forward, May

Sewell W. H. (1992). A Theory of Structure, Duality, Agency and Transformation. American Journal of Sociology, Vol. 98, No 1, pp1-29.Imperialism, Globalization and the Way Forward, May. http://dx.doi.org/10.1086/229967

Singh Ajit \& Dhumole Rahule (1999). The Global Merger Wave: Consequences for Developing Countries-Global Policy Forum, November.

Stallings Barbara (2001). Globalization and Liberalization: A view from the developing Countries United Nations Economic Commission for Latin America \& the Caribbean, February

Stiglitz, J. E. (2003). Globalization and its Discontents. W. W. Norton and Company Inc., New York.

Wade R. (1996), Globalization and Its Limits: Reports of the Death of the National Economy are exaggerated in Berger S. And Dore R. (eds), National Diversity and Global Capitalism, Cornell University Press.

Wolfensohn James (2001). Globalization Growth and Poverty: Building an inclusive World Economy. World Bank, December.

Yusuf S. (2001). Globalization and the Challenge for Developing Countries”, World Bank, June

\section{Copyright Disclaimer}

Copyright reserved by the author(s).

This article is an open-access article distributed under the terms and conditions of the Creative Commons Attribution license (http://creativecommons.org/licenses/by/3.0/). 